\title{
Grupo de convivência: percepção dos idosos em uma unidade básica de saúde
}

\author{
Socialization group: perception of elders in a primary health care unit
}

\author{
Grupo de convivencia: percepción de los ancianos en una unidad básica de salud
}

\author{
Recebido: 09/09/2017 \\ Aprovado: 05/06/2018 \\ Publicado: 01/08/2018
}

\author{
Ricardo Dias da Silva ${ }^{1}$
}

Este é um estudo exploratório, descritivo, de abordagem qualitativa, com o objetivo de avaliar a percepção dos idosos participantes de um grupo de convivência (GC) realizado em uma Unidade Básica de Saúde (UBS) localizada em uma comunidade rural da cidade de Patrocínio, situada no interior de Minas Gerais. 0 grupo existe desde o primeiro semestre de 2016 e a coleta de dados ocorreu entre abril e maio de 2017 na UBS. Entrevistou-se 5 mulheres participantes desde o início do GC com idade entre 60 a 79 anos; e, para a análise, utilizou-se o Discurso do Sujeito Coletivo (DSC).Obteve-se as Ideias Centrais: bom; comunicação; paciência; convivência; aprendizado e distração; muito importante.Evidenciou-se a importância da existência do GC com idosos na APS como: um espaço de socialização, local de escuta, desenvolvimento de habilidades, criação de ambiente saudável, meio para promoção da saúde e prevenção de agravos e doenças, lugar lúdico, melhora dos relacionamentos, tolerância, e estabelecimento de vínculo.

Descritores: Idoso; Educação em saúde; Promoção da saúde.

This is an exploratory, descriptive and qualitative study, aimed at evaluating the perception of elders who participate in a Socialization Group (SG) conducted in a Primary Health Care Unit (UBS) located in a rural community of the city of Patrocínio, MG, Brazil in the countryside of the Minas Gerais state. The group exists since the first semester of 2016, and data collection took place from April to May 2017, in the UBS. Five women were interviewed, all of which were participants of the SG since it started and aged from 60 to 79 years old. The analysis was conducted using the Discourse of the Collective Subject (DCS). The central ideas found were: good; communication; patience; socialization; learning and distraction; very important. The importance of the SG with elders in the Primary Health Care was made clear, as it was considered: a space for socialization, of listening, developing new skills, the creation of a healthy environment, a means to promote health and prevent diseases and health problems, a ludic place, an improvement of relationships, tolerance, and establishment of bonds.

Descriptors: Aged; Health education; Health promotion.

Este es un estudio exploratorio y descriptivo de abordaje cualitativo con el objetivo de evaluar la percepción de los ancianos participantes de un grupo de convivencia (GC) realizado en una Unidad Básica de Salud (UBS) localizada en una comunidad rural, de la ciudad de Patrocínio, MG, Brasil situada en el interior de Minas Gerais, Brasil. El grupo existe desde el primer semestre de 2016 y la colecta de datos ocurrió entre abril y mayo de 2017 en la UBS. Se entrevistaron a 5 mujeres participantes desde el inicio del GC con edad entre 60 y 79 años; y, para el análisis, se utilizó el Discurso del Sujeto Colectivo (DSC). Se obtuvieron las Ideas Centrales: bueno; comunicación; paciencia; convivencia; aprendizaje y distracción; muy importante. Se evidenció la importancia de la existencia del GC con ancianos en la APS como: un espacio de socialización, lugar de escucha, desarrollo de habilidades, creación de ambiente saludable, medio para promoción de la salud y prevención de agravamientos y enfermedades, lugar lúdico, mejoría de las relaciones, tolerancia y establecimiento de vínculo.

Descriptores: Anciano; Educación en salud; Promoción de la salud.

1. Enfermeiro. Especialista em Oncologia. Especialista em Saúde Coletiva. Especializando em Docência do Ensino Superior pela Universidade Cruzeiro do Sul. Mestrando em Atenção à Saúde pela Universidade Federal do Triângulo Mineiro. Enfermeiro Sanitarista da Secretaria Municipal de Saúde de Patrocínio, MG, Brasil. ORCID: 0000-0002-5856-9318 E-mail: ricardodiv@yahoo.com.br 


\section{INTRODUÇÃo}

$\mathbf{0}$ envelhecimento da população constitui um processo de proporção mundial que atinge também o Brasil e está relacionado aos processos de transição demográfica - caracterizada pelo aumento da expectativa de vida - e epidemiológica apresentando predominância das doenças crônicas não-transmissíveis $\quad(\mathrm{DCNT})^{1}$. Segundo o Instituto Brasileiro de Geografia e Estatística (IBGE), os idosos representam $11 \%$ da população brasileira, podendo chegar a 30 milhões de idosos em $2020^{2}$.

0 processo de envelhecimento provoca modificações fisiológicas, ocasionando maior vulnerabilidade a intercorrências patológicas nos aspectos social, biológico e psicológico (afetivo, emocional e cognitivo) ${ }^{3,4}$. Ações de promoção e educação em saúde centradas no autocuidado são necessárias à prevenção de agravos, incorporando estratégias de atenção social ao idoso ${ }^{1}$.

Nesse contexto, o grupo de convivência (GC) surge como uma importante contribuição para um envelhecimento saudável e com qualidade ${ }^{4}$. O GC pode representar um momento terapêutico para idosos, apoiando e fortalecendo o contexto social para o idoso $0^{5-7}$. 0 aprimoramento, o desenvolvimento de habilidades, a obtenção de mais conhecimentos para promoção da saúde (PS) e a melhoria na qualidade de vida (QV) são importantes ganhos que o GC pode oferecer.

Mais do que socializar o idoso, o GC pode oferecer uma nova identidade social ao mesmo $^{5}$. As atividades em grupo proporcionam a convivência e a prática de diversas atividades lúdicas, culturais e religiosas, além de promover vivências em processo grupal de formas diversificadas ${ }^{5}$. 0 compartilhamento na exploração das potencialidades promove o controle, a prevenção e o tratamento de possíveis transtornos psicossociais, dentre eles a depressão ${ }^{5}$.

A Atenção Básica (AB) consiste na porta de entrada preferencial do Sistema Único de Saúde (SUS) pelos usuários, sendo o ponto de partida para a estruturação dos sistemas locais de saúde; é representada por um conjunto de ações, individuais e coletivas, com atividades de promoção e proteção da saúde a serem desenvolvidas, visando a prevenção de agravos, diagnóstico, tratamento, reabilitação e a manutenção da saúde da população ${ }^{8}$.

Diante dessa realidade estabelecida no SUS, o GC de idosos é uma proposta que atua tanto na promoção da saúde, prevenção de doenças e agravos, tratamento e auxílio na recuperação do usuário com doenças crônicas de forma simultânea, como uma atividade integrativa, estimuladora e socializadora. Sendo assim, o GC é considerado um ambiente saudável, constituindo um dos parâmetros básicos para a PS apresentados na I Conferência Mundial sobre Promoção da Saúde, realizada no Canadá em 1986.

Em meio a esse cenário de aumento do envelhecimento da população brasileira e mundial, é relevante avaliar estratégias de abordagens que podem favorecer diretamente a qualidade de vida dos idosos. Este é um estudo que tem como objetivo avaliar a percepção dos idosos participantes de um grupo de convivência de uma Unidade Básica de Saúde (UBS) localizada em uma comunidade rural.

\section{MÉTODO}

Trata-se de um estudo exploratório, descritivo, de abordagem qualitativa com pesquisa de campo realizado na cidade de Patrocínio. O município é localizado no interior do Estado de Minas Gerais, na região do Triângulo Mineiro, tem cerca de 90.000 habitantes e Índice de Desenvolvimento Humano (IDH) avaliado em 0,799.

0 GC realizado na UBS foi criado em 30 de agosto de 2016 como uma ação promotora, preventiva, educativa e integradora. A ideia inicial das reuniões foi de ofertar uma estratégia de saúde mental - promotora de saúde, preventiva de doenças e agravos aos usuários adultos e idosos da UBS, posteriormente tornando-se um grupo de PS. A participação dos usuários no GC ficou sendo predominante por usuários idosos da comunidade.

A iniciativa, elaboração e a instituição do GC foi do enfermeiro coordenador da UBS. As reuniões são conduzidas pelo enfermeiro e 
ocorrem semanalmente (salvo em caso de imprevistos na agenda da UBS), sendo que ainda não houve a participação de outras categorias profissionais na condução do grupo.

Nos encontros são lidos textos, apresentadas músicas com o violão, realizadas terapias com silêncio e interiorização, exercícios de respiração, abordados temas relevantes sobre hábitos de vida saudáveis e, realizado debates sobre os assuntos escolhidos, e todos os integrantes do grupo são estimulados a participar expressando suas opiniões e ideias.

Dentre os temas que já foram abordados no GC realizado na UBS encontram-se: ansiedade; paz; perdão; empatia; calma; tranquilidade; simplicidade, expectativas futuras; motivação; alimentação saudável; lazer; benefícios da prática regular de atividade física, dentre outros.

Os temas são escolhidos para as reuniões de forma simples, clara e objetiva baseados nas características da população de abrangência e dos participantes do grupo, com o intuito de facilitar e estimular a participação dos usuários e proporcionar um momento de criação de vínculo, confiança e escuta do usuário.

Participaram do estudo cinco longevas, e a técnica de coleta de dados foi realizada mediante aplicação de entrevista semiestruturada, questionário sociodemográfico e questionário de variáveis clínicas realizadas entre os meses de abril e maio de 2017.

Foi realizada gravação em aparelho de áudio para posterior transcrição das falas, com prévia autorização das participantes. A média de tempo de cada entrevista foi de 10 minutos e o questionário sociodemográfico foi realizado na sequência da entrevista. Os parâmetros abordados na entrevista foram: "Qual é a sua percepção do grupo de convivência?"; "O que mudou em sua vida após a participação no grupo de convivência?"; "Você acha importante o grupo de convivência?".

As entrevistas foram analisadas a partir da modalidade de Discurso do Sujeito Coletivo (DSC). O DSC consiste em "um discurso síntese elaborado com pedaços de discursos de sentido semelhante reunidos num só discurso"10. O DSC é uma técnica utilizada em pesquisa qualitativa que tem como matériaprima os depoimentos escritos sobre vários discursos-síntese na primeira pessoa do singular ${ }^{11}$.

A modalidade do DSC consiste em selecionar de cada resposta individual coletada as Expressões-Chave (ECH), que são os trechos mais significativos destas respostas; as ECH correspondem as Ideias Centrais (IC) que são a síntese dos conteúdos expressos nas ECH. A partir dos dados das ECH e das IC, concebe-se os discursos-síntese, em primeira pessoa do singular, que são os DSC, e expressam o pensamento de um grupo como sendo um discurso individual ${ }^{11}$.

Os questionários sociodemográfico e de variáveis clínicas foram elaborados pelo pesquisador. No primeiro estão as variáveis: idade, gênero, cor da pele, renda, casa própria, número de pessoas que residem em casa (arranjo familiar), escolaridade e estado civil. Já no segundo questionário foi avaliado: presença de doenças crônicas não transmissíveis (DCNT), número de medicamentos em uso, quantidade de medicação psicotrópica atualmente prescrita, etilismo, tabagismo, sedentarismo e índice de massa corporal (IMC). Esses questionários tiveram a intenção de caracterizar a população participante do estudo.

A partir da interpretação dos dados coletados na entrevista semiestruturada, foram elaborados os DSC. Advindos do questionário sociodemográfico e de variáveis clínicas, dois quadros para explicitação dos dados obtidos foram desenvolvidos e processados. 0 delineamento do estudo com DSC e quadros foi feito utilizando-se os softwares Word $\AA$ e Excel $₫$, versões 2007.

Os critérios de inclusão foram: idade acima de 60 anos; ter participado de no mínimo 6 reuniões do grupo de convivência (GC) desde a sua criação; residir na área de abrangência da UBS; ter aceitado, mediante apreciação e assinatura de Termo de Consentimento Livre e Esclarecido (TCLE), participar do estudo. 
A pesquisa foi realizada mediante 0 esclarecimento prévio dos objetivos, métodos, critérios, riscos e benefícios aos participantes, além de assinatura do TCLE - elaborado e redigido pelo pesquisador dentro dos critérios da Resolução 466/2012 - em horário diferente do qual funciona a UBS e em local externo ao serviço de saúde.

\section{RESULTADOS}

Segundo questionário de avaliação sociodemográfica verificou-se que: todas as participantes eram do sexo feminino. Quanto à idade, tem-se 3 usuárias entre 60 e 69 anos e 2 entre 70 e 79 anos. Com relação à cor da pele foram 3 usuárias brancas e 2 pardas.

No parâmetro renda individual, foi obtido que as 5 usuárias têm 1 salário mínimo, casa própria, imóvel próprio, e escolaridade até o ensino primário.

Nas variáveis clínicas averiguou-se que, entre as DCNT encontradas, havia 4 usuárias com Hipertensão Arterial; 2 com Obesidade, 1 com Diabetes Mellitus, 1 com Doença Pulmonar Obstrutiva Crônica, 1 com Asma, 1 com Depressão, 1 com Artrose.

Para o número de medicações em uso encontrou-se: 1 em uso de um medicamento e 4 em uso de cinco medicamentos ou mais. Com relação à variável tabagismo e etilismo, apresentou-se que 2 usuárias são fumantes, e nenhuma etilista.

Dentre as cinco entrevistas realizadas com os idosos participantes do GC na UBS foram elaborados seis DSC, divididos nos seguintes temas: bom; comunicação; convivência; paciência; aprendizado e distração; muito importante. Para a pergunta sobre a percepção do grupo de convivência, compôs-se os DSC 1, 2 e 3, baseados nas IC: bom; comunicação; convivência. Os DSC são:

\section{IC1 / DSC 1: Bom}

Eu acho uma coisa boa. Acho muito bom. Eu acho bom participar, estou gostando. Sei que eu tenho que levantar e vir para o grupo que eu admiro. Felicidade pra todos nós que estamos aqui. Muito bom, muito legal. (I1, I2, I3, I4, I5).

IC2 / DSC 2: Comunicação

Todo mundo conversa, todo mundo expressa o que quer, fala. Às vezes a gente quase não tem contato e ali tem, a gente fala o que quer. A gente tem pouco diálogo em casa, e aqui gente conversa e pode distrair um pouco. (I1, I2, I3)

\section{IC 3 / DSC 3: Convivência}

As pessoas vão melhorando a convivência, o relacionamento. A gente tem contato com as colegas, com o instrutor. Estou gostando, uma dó não participar mais gente. $(I 2, I 3, I 5)$

De acordo com o questionamento feito às usuárias sobre as mudanças em sua vida após a participação no grupo de convivência elaborou-se o seguinte DSC, centrado nas IC: paciência; aprendizado; distração.

IC 4 / DSC 4: Paciência

Melhorou muita coisa, melhorou a saúde, a cabeça ta cansada, melhorou as preocupações. Eu sou muito impaciente, aprendi a ser mais calma. A gente tem mais tolerância, mais paciência. A gente fica feliz, tranqüila, calma, paciente. (I1, I2, I3, I4).

IC 5 / DSC 5: Aprendizado e distração

Melhorou muita coisa, mais entendimento. Melhorou umas coisinhas, coisas que a gente tá fazendo, coisas que eu não sabia e aprendi. A gente vai lá e passa o tempo, distrai, aprende. $(I 1, I 2, I 3, I 5)$

Com relação à questão sobre as usuárias acharem importante o grupo de convivência, pode-se observar o DSC relacionado na IC: muito importante.

IC 6 / DSC 6: muito importante.

Muito bom, gosto demais. Acho muito importante o grupo de convivência. Acho importante a convivência. Muito importante e uma dó não vir mais gente. (I1, I2, I3, I4, I5).

\section{DISCUSSÃO}

O aumento da população idosa no Brasil é evidente nas estatísticas do IBGE. Frente a esse cenário, fazem-se necessárias ações pertinentes no âmbito da PS, prevenção de doenças, busca da melhoria da QV e do atendimento humanizado na terceira idade.

O GC é considerado um importante espaço de socialização para a população idosa $^{5-7}$. Além disso, os GC proporcionam aos longevos suporte emocional, lazer, desenvolvimento de habilidades, aquisição de saberes, elevação da autoestima e ações de PS $^{5-7}$.

Os DSC relacionados à percepção das usuárias sobre o GC realizados nesse estudo mostram a necessidade de comunicação e da convivência em comunidade. Há por parte delas a necessidade de estar interagindo e de serem ouvidas, fazendo com que haja a assiduidade semanal no GC. A atividade grupal 
para idosos caracteriza-se um espaço de escuta ${ }^{13}$.

O GC é oportunidade de fazer e estreitar laços de amizades, sendo que os laços sociais vão sendo estimulados e se tornando interesantes ${ }^{5}$. Avalia-se que a segurança oferecida por um ambiente acolhedor e estimulante, como o do GC, é favorável para a autonomia do indivíduo na terceira idade ${ }^{14}$. 0 convívio com pessoas em condições iguais ou similares traz segurança e estímulo aos idosos no $\mathrm{GC}^{12}$.

Com relação ao questionamento sobre as mudanças proporcionadas pelo GC, percebe-se aumento na tolerância e paciência por parte das usuárias relacionadas a suas relações sociais e ao estímulo ao desenvolvimento e retorno das habilidades pessoais que elas relatam possuir. Como a QV está relacionada com a percepção do próprio indivíduo por si mesmo e associada ao meio social, infere-se que o GC interfere positivamente na melhoria da saúde psicológica, social e física dessas pessoas ${ }^{15}$.

As atividades realizadas no GC contribuem tanto para a manutenção do equilíbrio biopsicossocial do idoso e também para atenuar possíveis conflitos ambientais e pessoais $3,13,14$. As experiências e reflexões sobre o seu cotidiano estão entre os benefícios do $\mathrm{GC}^{15}$. As atividades grupais estimulam a socialização do idoso ${ }^{16}$.

0 desenvolvimento de habilidades pessoais é um dos ganhos do GC. As atividades que desenvolvem talentos estão dentre as propostas para exercer a PS de forma plena. A inserção de idosos em GC proporciona o conhecimento e compartilhamento de idéias $^{14}$.

$\mathrm{Na}$ visão geral das longevas, frente à indagação sobre a relevância do GC na UBS, observou-se que elas consideram as atividades grupais de muita importância. Infere-se que existe um considerável vínculo emocional entre as participantes, com as atividades que são realizadas e com o ambiente, que se tornou uma parte de suas rotinas e afazeres.

A identificação das usuárias com o GC, no âmbito da APS, é um fato vitorioso frente a um serviço de saúde público que ainda não rompeu definitivamente suas amarras do modelo biomédico ${ }^{17}$. Só com laços bem estreitos é possível realizar ações efetivas de PS. A prevenção de agravos e também a própria assistência ainda não alcançaram patamares impactantes; a promoção da saúde ainda é desconhecida para muitos ${ }^{17}$.

\section{CONCLUSÃO}

Visto que o envelhecimento da população traz consigo a necessidade de abordagens específicas, deve-se vencer o paradigma de enxergar erroneamente e de forma equivocada o envelhecimento como sendo uma fase de total dependência e ausência de socialização, sendo muitas vezes o que ocorre na sociedade.

Ao contrário, os serviços de saúde, profissionais, família e sociedade devem estimular e serem estimulados a uma ter visão das capacidades e das reais necessidades da população idosa no contexto atual, respeitando seus direitos e dignidade e atentos às transições demográfica e epidemiológica que ocorrem tardiamente no Brasil.

O grupo de convivência comprovou-se uma estratégia viável à Atenção Primária à Saúde no atendimento à terceira idade. Esta experiência sugere que aconteçam mais estudos sobre os grupos com idosos para ampliação dos conhecimentos nessa área e elaboração de novas abordagens de atenção à saúde dos longevos em todos os níveis de complexidade em saúde.

Dentre os benefícios do grupo de convivência observou-se o estabelecimento de relações, união, expressão de sentimentos, criação de vínculo com o serviço de saúde e melhoria do bem-estar.

Evidenciou-se a importância da existência do GC com idosos na APS como: um espaço de socialização, local de escuta, desenvolvimento de habilidades, criação de ambiente saudável, meio para promoção da saúde e prevenção de agravos e doenças, lugar lúdico, melhora dos relacionamentos, tolerância, e estabelecimento de vínculo. 


\section{REFERÊNCIAS}

1. Santos AS, Meneguci J, Silveira RE, Marquez FA, Ferreira VA, Viana DA. Perfil de saúde e qualidade de vida de idosos de Santa JulianaMG. Enferm Foco[Internet]. 2014[citado em: 20 abril 2017]; 5(3/4):61-4. DOI: http://dx.doi.org/10.21675/2357-

707X.2014.v5.n3/4.566

2. Instituto Brasileiro de Geografia e Estatística. Observações sobre a evolução da mortalidade no Brasil: o passado, o presente e perspectivas. Rio de Janeiro: IBGE; 2010.

3. Andrade AN, Nascimento MMP, Oliveira MMD, Queiroga RM, Fonseca FLA, Lacerda SNB, et al. Percepção de idosos sobre grupo de convivência: estudo na cidade de Cajazeiras-PB. Rev Bras Geriatr Gerontol. [Internet]. 2014 [citado em: 20 maio 2017]; 17(1):39-48.

http://dx.doi.org/10.1590/S1809-

98232014000100005

4. Ohara ECC, Ribeiro MP, Concone MHVB. Saúde do idoso. In: Ohara ECC, Saito RXS, organizadores. Saúde da família: considerações teóricas e aplicabilidade. $2 \mathrm{a}$ ed. São Paulo: Martinari; 2010. 480p.

5. Xavier LN, Sombra ICN, Gomes AMA, Oliveira GL, Aguiar CP, Sena RMC. Grupo de convivência de idosos: apoio psicossocial na promoção da saúde. Rev Rene [Internet]. 2015 [citado em: 22 abril 2017]; 16(4):557-66. Disponível em: http://www.revenf.bvs.br/pdf/rene/v16n4/ 1517-3852-rene-16-4-0557.pdf

6. Dalmolin IS, Leite MT, Hildebrandt, LM, Sassi MM, Perdossini LGB. A importância dos grupos de convivência como instrumento para a inserção social dos idosos. Rev Contexto Saúde [Internet]. 2011 [citado em: 23 abril 2017]; 10(20). Disponível em: https://www.revistas.unijui.edu.br/index.ph $\mathrm{p} /$ contextoesaude/article/view/1594

7. Wichmann FMA, Couto AN, Aerosa SVC, Mantañés MCM. Grupos de convivência como suporte ao idoso na melhoria da saúde. Rev Bras Geriatr Gerontol. [Internet] 2013 [citado em: 25 abril 2017]; 16(4):821-32. DOI: http://dx.doi.org/10.1590/S1809-

98232013000400016

8. Ministério da Saúde (Br). Secretaria de Atenção à Saúde, Departamento de Atenção
Básica. Política Nacional de Atenção Básica. Brasília: Ministério da Saúde; 2012. 110p.

9. Buss PM. Promoção da saúde e qualidade de vida. Ciênc Saúde Colet. [Internet]. 2000 [citado em: 25 abril 2017]; 1(5):163-77. DOI: http://dx.doi.org/10.1590/S1413-

81232000000100014

10. Figueiredo MZ, Chiari BM, Goulart BNG. Discurso do sujeito coletivo: uma breve introdução à ferramenta de pesquisa qualiquantitativa. Distúrb Comum. [Internet]. 2013 [citado em: 21 maio 2017]; 25(1). Disponível em: https://revistas.pucsp.br/index.php/dic/arti cle/view/14931

11. Lefevre F, Lefevre AMC. O sujeito coletivo que fala. Interface (Botucatu) [Internet]. 2006 [citado em: 13 maio 2017]; 10(20):517-24. DOI: http://dx.doi.org/10.1590/S141432832006000200017

12. Araújo JAT, Silva CSF, Silva BGM. A importância dos grupos de convivência para a saúde biopsicossocial dos idosos. In: IV Congresso Internacional de Envelhecimento Humano. UNICAP; Set-2010; Campina Grande, Brasil. Campina Grande, PB: Realize Eventos; 2010.

13. Casagrande GHJ, Silva MF, Carpes PMC. Qualidade de vida e incidência de depressão em idosas que frequentam grupos de terceira idade. Rev Bras Ciênc Envelhec Hum. 2013; 10(1):52-65.

14. Serbim AK, Figueiredo AEPL. Qualidade de vida de idosos em um grupo de convivência. [Internet]. Sci Med. (Porto Alegre). 2011 [citado em: 18 maio 2017]; 21(4):166-72. Disponível

em:

http://revistaseletronicas.pucrs.br/ojs/index .php/scientiamedica/article/download/9405 /7237

15. Silva, PAB. Fatores associados à qualidade de vida de idosos adscritos no Distrito Sanitário Noroeste de Belo Horizonte, Minas Gerais. [Dissertação]. Belo Horizonte: Universidade Federal de Belo Horizonte/UFMG; 2012. 184p.

16. Koch RF, Leite MT, Hildebrandt LM, Linck CL, Terra GT, Gonçalves LTH. Depressão na percepção de idosas de grupo de convivência. Rev Enferm UFPE. [Internet]. 2013 [citado em: 17 maio 2017]; 7(9):5574-82. Disponível 
em:

http://www.revista.ufpe.br/revistaenfermag em/index.php/revista/article/viewArticle/2 897

17. Santos AS. Aspectos da inserção de profissionais na atenção primária à saúde e o processo de formação [Editorial]. REFACS [Internet]. 2017 [citado em: 15 maio 2017];
5(2):179.

Disponível

em:

http://seer.uftm.edu.br/revistaeletronica/in dex.php/refacs/article/view/2091/2124

\section{CONTRIBUIÇÕES}

Ricardo Dias da Silva foi responsável por todas as etapas na execução deste estudo e confecção do artigo.

\section{Como citar este artigo (Vancouver)}

Silva RD. Grupo de convivência: percepção dos idosos em uma unidade básica de saúde. REFACS [Internet]. 2018 [citado em: inserir dia, mês e ano de acesso]; 6(3): 432-438. Disponível em: inserir link de acesso. DOI: inserir link do DOI.

\section{Como citar este artigo (ABNT)}

SILVA, R. D. Grupo de convivência: percepção dos idosos em uma unidade básica de saúde. REFACS, Uberaba, MG, v. 6, n. 3, p. 432-438, 2018. Disponível em: <inserir link de acesso>. Acesso em: inserir dia, mês e ano de acesso. DOI: inserir link do DOI.

Como citar este artigo (APA)

Silva, R. D. (2018). Grupo de convivência: percepção dos idosos em uma unidade básica de saúde. REFACS, 6(3), 432-438. Recuperado em inserir dia, mês e ano de acesso de inserir link de acesso. DOI: inserir link do DOI. 